\title{
Advanced Robot Design and Control Strategies for Human-Robot Cooperation
}

\author{
Gary M. Bone \\ McMaster University, Canada
}

\begin{abstract}
Robotic arms operating in close proximity with people are increasingly common in industrial applications (such as assembly) and non-industrial applications (such as assistive robots). The existing designs and control strategies used with these arms make a trade-off between the robot's safety and performance. For example, the arms designed for assistive robots are very safe, but can only carry small payloads at low speeds. Conversely, the arms designed for industry (known as "collaborative robots" or "cobots") can operate at high speeds with payloads exceeding $10 \mathrm{~kg}$, but with a greatly increased risk of serious human injuries. For example, the popular UR5 industrial robot has been shown to produce an impact force of over $1 \mathrm{kN}$. Can a robotic arm be simultaneously strong, fast and safe? This talk will seek an answer to that question.

Strategies to provide safety and performance can be categorized into three main areas: link design, actuator design, and actuator control. Approaches for improved link design include: use of compliant covers, lightweight materials and soft materials. Advanced actuation approaches include: electric motors with external torque sensing, series-elastic actuators, series clutch actuators, pneumatic artificial muscles and hybrid pneumatic-electric actuators. The influences of these approaches alone and in combination on the impact forces from simulated unconstrained and constrained head impacts are investigated and compared. Several model-based and non-model-based control strategies are studied. A conventional arm design actuated by DC motors with current limiting is used as the benchmark. The results demonstrate the advantages and disadvantages of each actuation and control approach. While no universal solution exists, the parallel-connected hybrid pneumatic-electric actuated arm employing model-based control produces the best combination of impact force reduction and trajectory tracking precision.
\end{abstract}

